\title{
EPR in B physics and elsewhere
}

\author{
Harry J. Lipkin ${ }^{a, b, c 1}$ \\ a Department of Particle Physics Weizmann Institute of Science, Rehovot 76100, Israel \\ b School of Physics and Astronomy, Raymond and Beverly Sackler Faculty of Exact \\ Sciences, Tel Aviv University, Tel Aviv, Israel
}

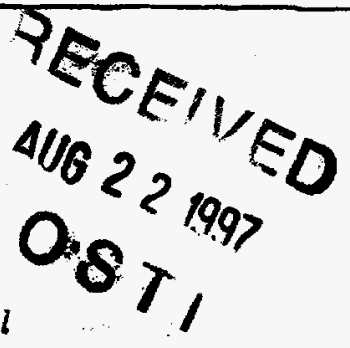

' High Energy Physics Division, Argonne National Laboratory, Argonne, IL 60439-4815, USA

\begin{abstract}
The application of Einstein-Podolsky-Rosen correlations in $\Upsilon(4 s) \rightarrow$ $B \bar{B}$ decays to research in CP violation is the first and probably only use of EPR as a technique for research in new physics. Elsewhere highly sophisticated EPR projects question EPR and test its predictions to look for violations of quantum mechanics, hidden variables, Bell's inequalities, etc.
\end{abstract}

In 1995 an international conference was held celebrating 60 years of EPR [1]. Nathan Rosen (the R of EPR) was still active and told us about Einstein's view of EPR. My paper on applications to kaon and B physics ( $\phi$ and B factories) [2] was the only talk on the program suggesting that EPR might be useful. All the others were on tests of quantum mechanics and included very beautiful photon interferometry experiments using the latest solid state technology to split a photon in a crystal into two photons with different energies and show that the split photons with only a piece of the original energy still exhibited the EPR correlations.

- Elsewhere: EPR was questioned and used to check Quantum Mechanics

- Hidden Variables, Bell's Inequality and Bohmian QM

- Sophisticated photon interferometry experiments confirm QM.

- B physics: EPR not only accepted but also crucial for BaBar.

- Lepton asymmetry observed in decay of one $B$ of pair from $\Upsilon(4 S)$ "entangled" with $\psi K_{\mathcal{S}}$ decay observed from other $B$.

1) Supported in part by grant No. I-0304-120-.07/93 from The German-Israeli Foundation for Scientific Research and Development and by the U.S. Department of Energy, Division of High Energy Physics, Contract W-31-109-ENG-38. 


\section{DISCLAIMER}

This report was prepared as an account of work sponsored by an agency of the United States Government. Neither the United States Government nor any agency thereof, nor any of their employees, makes any warranty, express or implied, or assumes any legal liability or responsibility for the accuracy, completeness, or usefulness of any information, apparatus, product, or process disclosed, or represents that its use would not infringe privately owned rights. Reference herein to any specific commercial product, process, or service by trade name, trademark, manufacturer, or otherwise does not necessarily constitute or imply its endorsement, recommendation, or favoring by the United States Government or any agency thereof. The views and opinions of authors expressed herein do not necessarily state or reflect those of the United States Government or any agency thereof. 


\section{DISCLAIMIER}

Portions of this document may be illegible in electronic image products. Images are produced from the best available original document. 
The problem of Entangled Wave functions

$$
\Psi_{(S=0)}=|-\vec{k}(\uparrow) ; \vec{k}(\downarrow)\rangle-|-\vec{k}(\downarrow) ; \vec{k}(\uparrow)\rangle=|-\vec{k}(\leftarrow) ; \vec{k}(\rightarrow)\rangle-|-\vec{k}(\rightarrow) ; \vec{k}(\leftarrow)\rangle
$$

Triggering on events with (Spin $\uparrow$ ) and momentum $-\vec{k}$ creates a polarized (Spin $\downarrow$ ) beam with momentum $\vec{k}$

But triggering on events with (Spin $\leftarrow$ ) and momentum $-\vec{k}$ creates a polarized (Spin $\rightarrow$ ) beam with momentum $\vec{k}$

- Elsewhere: How is this possible? How can the particle with momentum $\vec{k}$ know what is measured at momentum $-\vec{k}$ ? Something must be wrong with Quantum Mechanics!

- B physics: Great! We can make polarized beams with momentum $\vec{k}$ and arbitrary polarizations by choosing the right triggers with momentum $-\vec{k} !$

EPR and Entangled Wave functions in B physics at $\Upsilon(4 S)$ [3]

Define $B^{L}$ and $B^{B}$ as the linear combinations of $B^{\circ}$ and $\bar{B}^{\circ}$ that are mass eigenstates.

Both $B^{\circ}$ and $\bar{B}^{\circ}$ decay into $\psi K_{S}$.

Define $B^{1}$ and $B^{2}$ as just those particular linear combinations of $B^{\circ}$ and $\bar{B}^{\circ}$ such that $B^{1} \rightarrow \psi K_{S}$, but $B^{2}$ does not decay to $\psi K_{S}$; i.e. the $B^{\circ}$ and $\bar{B}^{\circ}$ contributions to $B^{2} \rightarrow \psi K_{S}$ exactly cancel one another.

$$
\Upsilon(4 S) \rightarrow\left|B_{-\vec{k}}^{\circ} ; \bar{B}_{\vec{k}}^{o}\right\rangle-\left|\bar{B}_{-\vec{k}}^{\circ} ; B_{\vec{k}}^{o}\right\rangle=\left|B_{-\vec{k}}^{R} ; B_{\vec{k}}^{L}\right\rangle-\left|B_{-\vec{k}}^{L} ; B_{\vec{k}}^{H}\right\rangle=\left|B_{-\vec{k}}^{1} ; B_{\vec{k}}^{2}\right\rangle-\left|B_{-\vec{k}}^{2} ; B_{\vec{k}}^{1}\right\rangle
$$

Triggering on events with $B^{\circ}$ decay observed with momentum $-\vec{k}$ creates a $\bar{B}^{\circ}$ beam with momentum $\vec{k}$

Triggering on events with $B^{B}$ observed with momentum $-\vec{k}$ creates a $B^{L}$ beam with momentum $\vec{k}$

Triggering on events with $B \rightarrow \psi K_{S}$ decay observed with momentum $-\vec{k}$ creates a $B^{2}$ beam with momentum $\vec{k}$

If $\mathrm{CP}$ is conserved, $B^{1}$ and $B^{2}$ are both mass eigenstates. Then $B^{2}$ decays have no time dependence; no lepton asymmetry.

If $C P$ is violated, $B^{1}$ and $B^{2}$ are not mass eigenstates. Time dependent lepton asymmetry oscillations can then be observed in $B^{2}$ decays.

"B Spin" and CP violation for Pedestrians [4]

Define " $B$ spin" eigenstates in which $B^{\circ}$ and $\bar{B}^{\circ}$ are states of spin-up and spin down in the $z$-direction of a fictitious $B$-spin space, the mass eigenstates $B^{B}$ and $B^{L}$ are up and down in the x-direction, and the state $B^{1} \rightarrow \psi K_{S}$ has its $B$ spin at some angle in the xy plane with respect to the $x$ axis. The angle is zero if CP is conserved and is simply related to one of the angles of the unitarity triangle in the standard model when $C P$ is violated. The states $B^{1}$ and $B^{2}$ precess around the $\mathrm{x}$-axis with a frequency given by the. $B^{B}-$ $B^{L}$ mass difference. All experiments are simply described as mesurements of polarizations and polarization correlations in this B spin space. 


\section{REFERENCES}

1. To be published as Proceedings of the Workshop 60 Years of EPR, Haifa (1995) in Annals of the Israel Physical Society.

2. Harry J. Lipkin, EPR in the 1990's, From Paradox to Crucial Ingredient in Frontier Experiments To be published as Proceedings of the Workshop 60 Years of EPR, Haifa (1995) in Annals of the Israel Physical Society.

3. Harry J. Lipkin, Physics Letters B219, (1989) 474

4. Harry J. Lipkin, In "Proceedings of the Workshop on b Physics at Hadron Accelerators" Snowmass, Colorado,June 21-July 3 (1993). edited by Patricia McBride and C. Shekhar Mishra, Published as SSCL-SR-1225 and FermilabCONF 93/267 (1993) p. 411 